\title{
Anestesia en enfermedades raras.
}

Errando-Oyonarte CL

Consorcio Hospital General Universitario de Valencia.

\section{Resumen}

Se presenta información acerca de la página web Orphananesthesia (www.orphananesthesia.eu), una base de datos del tratamiento anestésico de pacientes con enfermedades raras. Se hace énfasis en los aspectos que conlleva de seguridad para los pacientes y de información actualizada y compendiada, así como revisada por expertos, de fácil accesibilidad. La web estará disponible en breve en español, además de en inglés.

\section{Introducción}

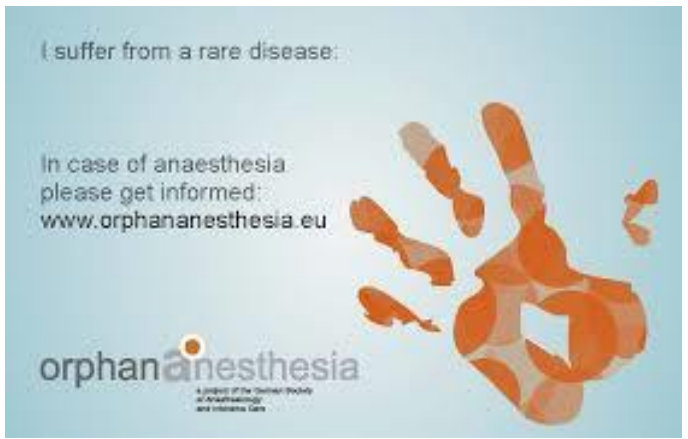

Se presenta información acerca de la página web: Orphananesthesia (https://www.orphananesthesia.eu/en/), una base de datos del tratamiento anestésico de pacientes con enfermedades raras. Se hace énfasis en los aspectos que conlleva de seguridad para los pacientes y de información actualizada y compendiada, así como revisada por expertos, de fácil accesibilidad. La web estará disponible en breve en español, además de en inglés.

\section{Sr. Editor de AnestesiaR:}

En 2004, el autor procedía a anestesiar a un paciente, de manera urgente, por hemoperitoneo debido a ruptura esplénica traumática, una situación relativamente habitual en la atención anestesiológica de urgencias. Solo que el paciente no "venía solo"; entre sus antecedentes figuraba una entidad denominada cardiomiopatía no compactante (1). No es objeto de esta carta describir el evento. El énfasis quería ponerlo en lo infrecuente de esta enfermedad y el hecho de que un anestesiólogo puede encontrarse de manera más o menos imprevista con un paciente que presenta una enfermedad rara o infrecuente y, quizá, no conocer cuál es el planteamiento más adecuado para el paciente concreto. De hecho, y solo a modo de ejemplo he podido anestesiar a diversos pacientes que las sufrían (2-6).

Emplearé el primer término, enfermedad rara, ya que es el más extendido.

En la práctica clínica de la anestesia (y cuidados intensivos y terapéutica del dolor), un médico especialista promedio puede llevar a cabo entre 400 y 500 procedimientos anestésicos al año. De entre todos estos pacientes muy pocos tendrán una enfermedad rara, y precisamente por ello sería del mayor 
interés disponer de información inmediata $\mathrm{y}$ actualizada sobre las entidades concretas. No es posible estar al día ni conocer los pormenores de estas enfermedades, que se definen como enfermedades con una incidencia global inferior a 5:10.000 (7). Es cierto que una búsqueda en alguno de los metabuscadores de internet (o en PubMed) puede suministrar información rápida, pero habitualmente será información parcial, o quizá poco fiable, inexistente o inconcreta. Desde 2005 el Grupo Científico de Trabajo de Anestesia Pediátrica de la Sociedad Alemana de Anestesiología y Cuidados intensivos que dirige el profesor Tino Münster, puso en marcha un proyecto que ha ido creciendo poco a poco. Se trata del Proyecto denominado "Orphananesthesia“. El objeto del mismo fue "crear una base de datos abierta con información sobre la anestesia de pacientes con enfermedades raras"

(www.orphananesthesia.eu). Dicha base de datos contiene hasta el momento información específica de unas 180 enfermedades, en inglés y parcialmente en alemán. El motivo de comunicarlo aquí es difundir su existencia y poner en conocimiento de los anestesiólogos que la web completa ha sido traducida al español, incluidas hasta el momento unas 100 enfermedades. También se está traduciendo a otros idiomas (italiano, portugués, francés). La traducción se ha llevado a cabo por un grupo de anestesiólogos del Hospital General de Valencia y otros, pero está abierta a la colaboración de cualquiera que tenga información relevante sobre enfermedades raras y su tratamiento anestésico o de cuidados postoperatorios, críticos e intensivos. Las formas de colaboración son diversas

(ver www.orphananesthesia.eu): hacer una revisión a partir de un caso clínico que se haya tenido ocasión de tratar, se puede sugerir una enfermedad rara para llevar a cabo la revisión, hacer la propia revisión de la entidad (no solo casos clínicos), sugerir autores que puedan tener experiencia, presentar iconografía, hacer traducciones al español de las ya publicadas, etc. Para ello los interesados pueden contactar con el Editor español (Dr. Carlos L. Errando -el autorerrando013@gmail.com) o bien a través deinfo@orphananesthesia.eu (en inglés o alemán).

La colaboración se lleva a cabo de manera desinteresada y solo cuenta con financiación y apoyo para la web de la Sociedad alemana de anestesia.

La denominada "ficha de enfermedad" consta de un Resumen con datos genéticos y clínicos, la cirugía o procesos más habituales a los que los pacientes pueden ser sometidos, los tipos de anestesia sugeridos, si precisan evaluación preoperatoria adicional a la estándar, peculiaridades de la vía aérea, transfusión / coagulación, anticoagulación, posicionamiento / transporte, interacciones medicamentosas, particularidades de la anestesia y monitorización, complicaciones previsibles. También cuidados postoperatorios, diagnóstico diferencial de eventos y si es factible anestesia ambulatoria y anestesia obstétrica. Finalmente bibliografía y enlaces de internet de interés.

Como criterio de calidad, todas las revisiones son evaluadas por expertos (peer review), al menos dos, un anestesiólogo y un especialista en la enfermedad de que se trata (genetista, internista, pediatra, neurólogo, etc.). Los artículos también se publican en inglés en un suplemento mensual de la Revista alemana de anestesiología y medicina intensiva (8).

Volviendo al motivo clínico del interés del proyecto, varios aspectos lo enfatizan aún más: (a) hay muchísima 
información genética, médica, de aspectos pronósticos, terapéuticos, de asociaciones de expertos y de pacientes (ver www.orpha.net), pero escasa del tratamiento anestésico, (b) a pesar de la rareza de las enfermedades, la tendencia es a encontrar casos en el quirófano o en salas de intervencionismo con frecuencia creciente. Esto es debido, en parte, a que la supervivencia de estos pacientes es mayor, o en el caso de mujeres a que éstas llegan a la edad fértil, etc, y finalmente, (c) un aspecto de suma importancia, que resume todos los anteriores, es la seguridad tanto del paciente como del profesional.

Nuestro interés, que se inició con la traducción de los artículos, se ha ampliado con el tiempo puesto que el feed-back que nos hacen llegar desde Orphananesthesia indica que los accesos desde España y los países de habla hispana es creciente, y, por tanto, intentaremos que el servicio de la web a la comunidad anestesiológica vaya en aumento; para ello queremos contar con la colaboración de todos los anestesiólogos. De hecho, los objetivos principales de Orphananesthesia son publicar las recomendaciones y su difusión.

\section{Bibliografía}

- Errando CL, Tatay J, Serrano-Romero A, Gudín-Uriel M, Revert M, Peiró CM. Splenic rupture and haemoperitoneum in a patient diagnosed of noncompaction of the left ventricular myocardium. Br J Anaesth 2005, 95(3):358-361. (․ㅡ)

- Errando CL. Comments on a case report of Angelman syndrome anaesthesia. Anaesthesia 2008; 63(10): 1145-1146. (HTML)
- Errando CL, Navarro L, Vila M, Pallardó MA. Anesthetic management of a patient diagnosed with CADASIL (cerebral arteriopathy, autosomal dominant, with subcortical infarcts and leukoencephalopathy). Rev Esp Anestesiol Reanim 2012; 59(2): 102-106. (ubMed)

- Errando CL, Pérez-Caballero P. Comments on the "Anaesthetic management in patients with Duchenne muscular dystrophy". Eur J Anaesthesiol 2013; 30(5): 257. doi: 10.1097/EJA.0b013e32835d84ed. (HTML)

- Gálvez-Cañellas JL, Errando CL, MartínezTorrente F, Mayor F, Zasadowski M, Villanueva Y, Soria-Bretones C. Anesthesia for orphan diseases. Difficult monitoring of neuromuscular blockade in a patient with severe Charcot-Marie-Tooth disease type I. Eur J Anaesthesiol 2013; 30 (12): 772-775. doi: 10.1097/EJA.0b013e3283623dea. (HTML)

- Gálvez-Cañellas JL, Errando CL, Ordóñez Arana A, Falcó E, Mazzinari G, Robles D, Vila Sánchez M. Eficacia de sugammadex para la reversión del bloqueo neuromuscular en una mujer con distrofia muscular de Becker. Rev Esp Anestesiol Reanim 2011; 58(10): 614-617. (Medes)

- $\quad$ https://www.orphananesthesia.eu/en/theproject/faq.html

- Errando CL. CADASIL syndrome. Anästh Intensivmed 2014;55:S506-S524. (HTML)

Correspondencia al autor

Carlos Luis Errando-Oyonarte errando013@gmail.com

FEA. Servicio de Anestesiología, Reanimación y Terapéutica del Dolor.

Consorcio Hospital General Universitario de Valencia

Aceptado para el blog en marzo de 2020 